\title{
Synergistic Anti-SARS-CoV-2 Activity of Repurposed Anti-Parasitic Drug Combinations
}

\section{Kunlakanya Jitobaom}

Mahidol University Faculty of Medicine Siriraj Hospital

\section{Chompunuch Boonarkart}

Mahidol University Faculty of Medicine Siriraj Hospital

\section{Suwimon Manopwisedjaroen}

Mahidol University Faculty of Science

\section{Nuntaya Punyadee}

Mahidol University Faculty of Medicine Siriraj Hospital

\section{Suparerk Borwornpinyo}

Mahidol University Faculty of Science

Arunee Thitithanyanont

Mahidol University Faculty of Science

\section{Panisadee Avirutnan}

Mahidol University Faculty of Medicine Siriraj Hospital

Prasert Auewarakul ( $\nabla$ prasert.aue@mahidol.ac.th )

Mahidol University Faculty of Medicine Siriraj Hospital https://orcid.org/0000-0002-4745-4291

\section{Research Article}

Keywords: SARS-CoV-2, repurposed drug, anti-parasitic drugs, Niclosamide, Ivermectin, Chloroquine

Posted Date: November 29th, 2021

DOI: https://doi.org/10.21203/rs.3.rs-1069947/v1

License: (c) (i) This work is licensed under a Creative Commons Attribution 4.0 International License. Read Full License 


\section{Abstract}

COVID-19 pandemic has claimed millions of lives and devastated the health service system, livelihood and economy in many countries worldwide. Despite the initiation of vaccination programs in many countries, the spread of the pandemic continues and effective treatment is still urgently needed. Although some antiviral drugs have been shown to be effective, they are not widely available. Repurposing of antiparasitic drugs with in vitro anti-SARS-CoV-2 activity is a promising approach being tested in many clinical trials. Combination of these drugs is a plausible way to enhance their effectiveness. We tested in vitro anti-SARS-CoV-2 activity of combinations of Niclosamide, Ivermectin, and Chloroquine; and show here that these combinations resulted in more than 10 -fold reduction in the half maximal inhibitory concentration $\left(\mathrm{IC}_{50}\right)$ as compared to individual drugs. Synergy landscape analyses showed NiclosamideIvermectin combination to have the best synergy score with a peak Loewe synergy score of over 20 and a mean score of 6.60 in Vero E6 cell and a peak Loewe synergy score of 13.2 and a mean score of 2.897 in Calu-3 cells.

\section{Introduction}

The spread of SARS-CoV-2 and the COVID-19 pandemic has swept through countries and continents causing catastrophic loss to lives, public health, livelihood, and economy. Up to March 2021, more than hundred million cases have been reported with over two million deaths [2]. The hope to get through the pandemic and resume normal life relies heavily on vaccine deployment, which will still take months or years in most less-developed countries. One of the reasons for the heavy loss of lives, hospital overload, and public panic is the lack of effective treatment. Remdesivir is now the only antiviral drug with emergency use authorization by US FDA [39]. The drug is, however, not yet widely available. Other FDAapproved drugs are anti-inflammatory targeting host inflammatory responses [38]. More drugs capable of inhibiting SARS-CoV-2 replication are urgently needed not only for treatment but also for reducing viral load and transmission. Many repurposed anti-parasitic drugs have been shown to possess in vitro activity against SARS-CoV-2.

In vitro screenings of FDA-approved drugs have identified a number of anti-parasitic drugs with antiSARS-CoV-2 activity and potential for drug repurposing for treatment of COVID-19 patients $[4,20]$. The early hope to get an effective treatment using these drugs was let down by the failure to show clinical benefit of Chloroquine in clinical trials [35]. On the other hand, Ivermectin has shown promising results in many clinical trials $[1,6,10-12,17,25,40]$. Ivermectin has been shown to cause up to 5000 -fold reduction in SARS-CoV-2 replication in vitro $[9,13,19]$. The drug has been widely used to treat various parasitic diseases in humans and animals for four decades with little safety concern. It was also used in the mass treatment campaign against river blindness (Onchocerciasis) with good safety record [32]. It is therefore, an attractive option for drug repurposing for COVID-19 treatment. Another anti-parasitic drug, Niclosamide, showed a good anti-SARS-CoV-2 activity with a high selective index $[20,28]$. The drug has been shown to exhibit broad antiviral activity against a wide range of viruses [45]. These anti-parasitic drugs with potent in vitro anti-SARS-CoV-2 activity are widely available, inexpensive, and considered 
relatively safe for short-term usage. They were therefore selected for synergistic testing in order to find combination regimens with good potential for drug repurposing in COVID-19 treatment. The world urgently needs repurposed drug regimens with higher anti-SARS-CoV-2 activity in order to cope with the pandemic. An approach to enhance drug potency is through drug combination.

\section{Materials And Methods Chemicals}

The $10 \mathrm{mM}$ stock solutions were prepared in culture-grade 100\% DMSO (Sigma) for Niclosamide (N3510, Sigma), and Ivermectin (18898, Sigma), or water for Chloroquine (HY-17589, MCE) and stored at $-80^{\circ} \mathrm{C}$. All drugs were diluted to the working concentrations in $2 \%$ FBS-MEM or $2 \% F B S-D M E M / F 12$ for the treatments in Vero E6 and Calu-3 cells, respectively. The final concentration of DMSO was $0.5 \%$ in all experiments.

\section{Cells And Viruses}

Vero E6 (CRL-1586, ATCC) cells were cultivated in the minimum essential medium (MEM; 10-009-CV, Corning) supplemented with $10 \%$ heat inactivated FBS (10\%FBS-MEM) at $37^{\circ} \mathrm{C}$ with $5 \% \mathrm{CO}_{2}$. Calu- 3 cells (HTB-55, ATCC) were cultivated in Dulbecco's Modified Eagle Medium/Nutrient Mixture F-12 (DMEM/F-12; 11320033 , Gibco) supplemented with $10 \%$ heat inactivated FBS (10\%FBS-DMEM/F12) at $37^{\circ} \mathrm{C}$ with $5 \%$ $\mathrm{CO}_{2}$.

SARS-CoV-2 (SARS-CoV-2/01/human/Jan2020/Thailand) was previously isolated from nasopharyngeal swabs of a COVID-19 case in Thailand [22]. The virus was propagated in Vero E6 cells. The supernatants containing virus were harvested by centrifugation to remove cell debris, then aliquot and stored at $-80^{\circ} \mathrm{C}$. The viral titer was determined by plaque assay or $50 \%$ tissue culture infectious dose $\left(\mathrm{TCID}_{50}\right)$ endpoint dilution assay.

\section{Virus Infection}

Vero E6 or Calu-3 cells were seeded in culture plates at a density that allowed $100 \%$ and $70 \%$ confluence to be reach, respectively. The cell culture supernatants were removed and the cells were incubated with $2 \%$ FBS-MEM or $2 \%$ FBS-DMEM/F12 containing SARS-CoV-2 at the indicated multiplicity of infection (m.o.i.) or $2 \% \mathrm{FBS}$-media as a mock infection for one hour at $37^{\circ} \mathrm{C}$ with $5 \% \mathrm{CO} 2$. Subsequently, the viral inoculum was removed, and the cells were maintained in 2\%FBS-MEM or 2\%FBS-DMEM/F12 for Vero E6 or Calu-3 cells, respectively, for the indicated time periods.

\section{Viral Titration}




\section{Plaque assay}

Vero E6 cells were plated in 24-well plates at a density of $1.3 \times 10^{5}$ cells per well before the day of infection. Then the culture medium was removed, and the cells were inoculated with 10 -fold serial dilution of virus supernatants for one hour at $37^{\circ} \mathrm{C}$ with $5 \% \mathrm{CO}_{2}$. Subsequently, the virus supernatants were removed, and the cells were overlaid with $1 \mathrm{ml}$ of $1.56 \%$ microcrystalline cellulose (Avicel, RC-591) in $2 \%$ FBS-MEM. The cells were incubated at $37^{\circ} \mathrm{C}$ with $5 \% \mathrm{CO}_{2}$ for three days. The overlaid medium was removed, and the cells were fixed with 10\% (v/v) formalin in phosphate-buffered saline (PBS) for $2 \mathrm{hr}$. The fixed infected cells were washed in tap water and stained with $1 \%(\mathrm{w} / \mathrm{v})$ crystal violet in $20 \%(\mathrm{v} / \mathrm{v})$ ethanol for $5 \mathrm{~min}$ and washed to remove the excess dye. The plaques were counted and the viral titers were calculated in plaque forming units per $\mathrm{ml}(\mathrm{pfu} / \mathrm{ml})$.

\section{Tcid Endpoint Dilution Assay}

Calu-3 cells were seeded in 96 -well plates at a density of $2.5 \times 10^{4}$ cells/well. The culture medium was removed, and the cells were incubated with half-log 10 serial dilution of the virus stock for 2 days at $37^{\circ} \mathrm{C}$ with $5 \% \mathrm{CO}_{2}$. After that, the cells were fixed with $1: 1$ methanol/acetone for $30 \mathrm{~min}$ at $4{ }^{\circ} \mathrm{C}$ and the infectivity was detected with an antibody against the SARS-CoV-2 nucleocapsid protein (40143-R001, Sino Biological) and the appropriate secondary antibody-congugated HRP. The viral $\operatorname{TCID}_{50}$ titers were calculated using the Reed and Muench method [31].

\section{One-step Quantitative Reverse-transcription Pcr (Qrt-pcr)}

The one-step qRT-PCR was used as a screening assay to detect the RNA of SARS-CoV-2 directly from the virus supernatants, without RNA purification [14]. Virus supernatants were heat inactivated at $70^{\circ} \mathrm{C}$ for 20 min and diluted with DNase/RNase free distilled water for a ratio of 1:10. Subsequently, one-step qRT-PCR was performed using the Power SYBR one-step kit (Applied Biosystems) and the LightCycler 480 (Roche, LC480). The one-step RT-PCR master mix was prepared following the kit's instructions for a $10 \mu$ l reaction volume.

The primers used were CCDC-N-Fw: 5'-GGGGAACTTCTCCTGCTAGAAT-3'and CCDC-N-Rv: 5'-

CAGACATTTTGCTCTCAAGCTG-3'. The master mix was mixed with $4.6 \mu$ of the diluted sample in $96-$ well white PCR plate. RNA of SARS-CoV-2 purifying from the virus stock using TRIzol-LS (Invitrogen) was used as a positive control. The samples also include no-template control (nuclease-free water and the medium of mock infected cells). The LC480 was run according to the Power SYBR one-step kit's instructions. For briefly, the revere transcription step at $48^{\circ} \mathrm{C}$ for $30 \mathrm{~min}$ and the activation of polymerase at $95^{\circ} \mathrm{C}$ for 10 min. Then followed by 45 amplification cycles $\left(95^{\circ} \mathrm{C}\right.$ for $15 \mathrm{~s}, 60^{\circ} \mathrm{C}$ for $\left.1 \mathrm{~min}\right)$ and melt curve step to 
determine the specificity of the PCR product from the melting temperature $\left(T_{m}\right)\left(95^{\circ} \mathrm{C}\right.$ for $30 \mathrm{~s}, 60^{\circ} \mathrm{C}$ for 30s).

The threshold cycle $(\mathrm{Ct}$ ) values were calculated from raw fluorescence data using Abs Quant/2nd derivative method. The $T_{m}$ calling analysis was performed to exclude reactions with non-specific amplification by comparing with the product amplified from positive control and the no template control. The inhibition of SARS-CoV-2 production in drug-treated cells was relative to the cells treated with $0.5 \%$ DMSO-2\%FBS-MEM.

\section{Cell Viability Assay}

Vero E6 or Calu-3 cells were seeded in 96 well-plates at a density that allowed $100 \%$ and $70 \%$ confluence to be reached, respectively. The culture medium was removed, then various concentrations of drugs in $2 \%$ FBS-MEM or $2 \%$ FBS-DMEM/F12 were added to the cells for 48 hours. After that, the cell viability was assessed using MTT dye (Invitrogen). The viable cells would convert the 3-(4, 5-dimethyl-2-thiazolyl)-2, 5diphenyl-2H-tetrazolium bromide to MTT formazan. The precipitates of MTT formazan in the cells then were dissolved by DMSO. The absorbance was measured at $570 \mathrm{~nm}$. The cells treated with $0.5 \%$ DMSO were used as a control ( $100 \%$ cell viability).

Evaluations of antiviral activity against SARS-CoV-2 in vitro

\section{Single Drug Treatments}

Vero E6 or Calu-3 cells were seeded in 96 well-plates at a density of $2.5 \times 10^{4}$ or $2.0 \times 10^{4}$ cells per well, respectively. The drugs were serially diluted by twofold in $2 \%$ FBS-media. Then the cells were incubated with the serial dilution drugs or no drug control $(0.5 \% \mathrm{DMSO})$ for one hour at $37^{\circ} \mathrm{C}$ with $5 \% \mathrm{CO}_{2}$. Subsequently, the virus at m.o.i. 0.01 or $500 \mathrm{TCID}_{50} / 100 \mu \mathrm{l}$ was added to Vero E6 or Calu-3 cells, respectively, and incubated for one hour. After that, the mixtures of drug and virus were removed and the cells were further maintained in the $2 \%$ FBS-media containing the serial dilution drugs or $0.5 \%$ DMSO for 48 hours. The culture supernatants were collected and the viral titers were determined using a plaque assay and one-step qRT-PCR.

\section{Two-drug Combinations Treatments}

Vero E6 or Calu-3 cells were seeded in 96 well-plates at a density of $2.5 \times 10^{4}$ or $2.0 \times 10^{4}$ cells per well, respectively. The cells were treated for one hour with 16 different pairwise combinations of two drugs. The drug concentrations ranged between $2 \times, 1 \times, 0.5 \times$ and $0.25 \times$ of $I_{50}$ values. Subsequently, the cells were infected with SARS-CoV-2 following the same approach used in single drug treatment. The virus supernatants were collected for titration by qRT-PCR or a plaque assay. 


\section{The Combination Synergy Analysis}

The SynergyFinder web application was used to analyze and visualize the degree of combination synergy between two drugs. The synergy scores of two-drug combinations were analyzed by comparing the observed drug combination response (percent inhibition) against the expected response, calculated using a reference model [18]. Four reference models were used in this study, including the Loewe additivity (Loewe), Zero Independence Potency (ZIP), Highest Single Agent (HSA), and Bliss independence models [26].

\section{Statistical analysis}

The independence experiments were performed in triplicated, and data are shown as mean \pm SD. The $50 \%$ cytotoxic concentration $\left(\mathrm{CC}_{50}\right)$ and the half-maximal inhibitory concentration $\left(\mathrm{IC}_{50}\right)$ were calculated from the dose-response curves of drug treatment against SARS-CoV-2 by non-linear regression analysis using GraphPad Prism 8 (GraphPad Software, Inc., CA).

\section{Results}

\section{Evaluation of single drug treatment against SARS-CoV-2 in Vero E6 cells}

Figure 1 and Table 1 show the anti-SARS-CoV-2 activities and cytotoxicity of the repurposed drugs in Vero E6 cells. The plaque assay was used to determine the viral production and is expressed as the percent inhibition relative to the viral titer of DMSO-treated cells. The one-step qRT-PCR was used to quantitate the viral RNA in virus supernatants and is also expressed as the percent inhibition relative to the DMSOtreated cells. The $\mathrm{IC}_{50}$ values calculated from the dose-response determined by plaque assay for Niclosamide, Ivermectin, and Chloroquine were $0.049,1.23,0.046$ and $0.83 \mu \mathrm{M}$, respectively. The $\mathrm{IC}_{50}$ values calculated from the dose-response determined by one-step qRT-PCR for Niclosamide, Ivermectin, and Chloroquine were $0.043,1.27$, and $0.89 \mu \mathrm{M}$, respectively. Both methods used for viral quantification resulted in similar IC $\mathrm{C}_{50}$ values. Thus the viral RNA quantification by the one-step qRT-PCR accurately determined the infectious virus output in these experiments, and could be used for the further two-drug combination experiments for the high throughput screening. 
Table 1

Single drug treatment against SARS-CoV-2 in vitro.

\begin{tabular}{|c|c|c|c|c|c|}
\hline \multirow[t]{2}{*}{$\begin{array}{l}\text { Drug } \\
\text { candidates }\end{array}$} & Drug class & Drug indication & $\begin{array}{l}\mathrm{CC}_{50} \\
(\mu \mathrm{M})\end{array}$ & $\begin{array}{l}I_{50} \\
(\mu M)\end{array}$ & $\begin{array}{l}I_{50} \\
(\mu M)\end{array}$ \\
\hline & & & & $\begin{array}{l}\text { Plaque } \\
\text { assay }\end{array}$ & $\begin{array}{l}\text { qRT- } \\
\text { PCR }\end{array}$ \\
\hline \multirow[t]{2}{*}{ Niclosamide } & $\begin{array}{l}\text { Anthelminthic } \\
\text { agents }\end{array}$ & $\begin{array}{l}\text { Treatment of tapeworm and intestinal } \\
\text { fluke infections }\end{array}$ & 0.29 & 0.049 & 0.043 \\
\hline & & [13] & & & \\
\hline Ivermectin & $\begin{array}{l}\text { Anti-parasitic } \\
\text { agents }\end{array}$ & $\begin{array}{l}\text { Treatment of onchocerciasis, and } \\
\text { other worm infestations [14] }\end{array}$ & 10.55 & 1.23 & 1.27 \\
\hline Chloroquine & $\begin{array}{l}\text { Anti-malarial } \\
\text { agents }\end{array}$ & $\begin{array}{l}\text { Treatment of malaria, rheumatic } \\
\text { diseases and Zika virus infection [50] }\end{array}$ & 118.20 & 0.83 & 0.89 \\
\hline
\end{tabular}

\section{Evaluation of two-drug combination treatments against SARS-CoV-2 in Vero E6 cells}

Firstly, the antiviral activities of two-drug combinations were assessed in vitro in Vero E6 cells. The cells were treated with 16 different pairwise combinations of two drugs, including, Niclosamide-lvermectin, Niclosamide-Chloroquine and Ivermectin-chloroquine.

\section{Niclosamide-ivermectin Combination}

The presence of Ivermectin induced a shift in the dose-response curve of Niclosamide, with 10.75-fold reduction of Niclosamide $\mathrm{IC}_{50}$ value in the presence of 2.4 and $1.2 \mu \mathrm{M}$ Ivermectin and approximately 2fold reduction of Niclosamide $\mathrm{IC}_{50}$ value in the presence of 0.6 and $0.3 \mu \mathrm{M}$ Ivermectin (Fig. 2A, Table 2). In a similar way, the presence of Niclosamide also induced a shift in Ivermectin dose-response curve, with 26.46-fold reduction of Ivermectin $\mathrm{IC}_{50}$ value in the presence of $0.09 \mu \mathrm{M}$ Niclosamide (Fig. 2B, Table 2). The presence of $0.045 \mu \mathrm{M}, 0.0225 \mu \mathrm{M}$ and $0.01125 \mu \mathrm{M}$ Niclosamide resulted in 7.18, 4.06 and 1.92-fold reduction of Ivermectin $\mathrm{IC}_{50}$ value, respectively. The dose-response matrix of Niclosamide and Ivermectin combination showed the obvious increasing inhibitory effects (Fig. 2C). A synergy landscape plot showed high positive Loewe synergy scores in combinations with Ivermectin concentration higher than $0.6 \mu \mathrm{M}$ with a peak score of 22.76 indicating a synergistic effect. The scores were low positive to slightly negative in the other part of the plot with lower Ivermectin concentration indicating only additive effect at these low concentrations (Fig. 2D). The mean Loewe synergy score is 6.60. The ZIP, Bliss independence and HSA reference models were also used, the results showed the synergy scores of 12.64, 12.77 and 19.03 , respectively, which accounted for the synergistic effect between Niclosamide and Ivermectin in Vero E6. No significant cytotoxicity in all 16 pairwise combinations (Fig. 2A, B). 
Table 2

Antiviral activity of two-drug combinations treatment against SARS-CoV-2 in Vero E6 cells.

\begin{tabular}{|c|c|c|c|}
\hline \multicolumn{2}{|l|}{ Drug treatment } & \multirow{2}{*}{$\begin{array}{l}\mathrm{IC}_{50} \\
(\mu \mathrm{M}) \\
\text { qRT- } \\
\text { PCR }\end{array}$} & \multirow{2}{*}{$\begin{array}{l}\text { Fold reduction of } \mathrm{IC}_{50} \\
\text { (single/combined) }\end{array}$} \\
\hline & & & \\
\hline \multirow{10}{*}{$\begin{array}{l}\text { Niclosamide- } \\
\text { Ivermectin }\end{array}$} & Niclosamide & 0.043 & \\
\hline & $\begin{array}{l}\text { Niclosamide + Ivermectin } 2.4 \\
\mu \mathrm{M}\end{array}$ & 0.004 & 10.750 \\
\hline & $\begin{array}{l}\text { Niclosamide + Ivermectin } 1.2 \\
\mu \mathrm{M}\end{array}$ & 0.004 & 10.750 \\
\hline & $\begin{array}{l}\text { Niclosamide + Ivermectin } 0.6 \\
\mu \mathrm{M}\end{array}$ & 0.018 & 2.399 \\
\hline & $\begin{array}{l}\text { Niclosamide + Ivermectin } 0.3 \\
\mu \mathrm{M}\end{array}$ & 0.022 & 1.955 \\
\hline & Ivermectin & 1.27 & \\
\hline & $\begin{array}{l}\text { Ivermectin + Niclosamide } 0.09 \\
\mu \mathrm{M}\end{array}$ & 0.048 & 26.46 \\
\hline & $\begin{array}{l}\text { Ivermectin + Niclosamide } \\
0.0045 \mu \mathrm{M}\end{array}$ & 0.177 & 7.18 \\
\hline & $\begin{array}{l}\text { Ivermectin + Niclosamide } \\
0.0225 \mu \mathrm{M}\end{array}$ & 0.313 & 4.06 \\
\hline & $\begin{array}{l}\text { Ivermectin + Niclosamide } \\
0.01125 \mu \mathrm{M}\end{array}$ & 0.660 & 1.92 \\
\hline \multirow{7}{*}{$\begin{array}{l}\text { Niclosamide- } \\
\text { Chloroquine }\end{array}$} & Niclosamide & 0.043 & \\
\hline & $\begin{array}{l}\text { Niclosamide + Chloroquine } 1.7 \\
\mu \mathrm{M}\end{array}$ & 0.003 & 14.333 \\
\hline & $\begin{array}{l}\text { Niclosamide + Chloroquine } 0.85 \\
\mu \mathrm{M}\end{array}$ & 0.009 & 4.778 \\
\hline & $\begin{array}{l}\text { Niclosamide + Chloroquine } \\
0.425 \mu \mathrm{M}\end{array}$ & 0.013 & 3.308 \\
\hline & $\begin{array}{l}\text { Niclosamide + Chloroquine } \\
0.2125 \mu \mathrm{M}\end{array}$ & 0.029 & 1.483 \\
\hline & Chloroquine & 0.89 & \\
\hline & $\begin{array}{l}\text { Chloroquine + Niclosamide } 0.09 \\
\mu \mathrm{M}\end{array}$ & 0.028 & 31.78 \\
\hline
\end{tabular}




\begin{tabular}{|c|c|c|c|}
\hline \multirow[t]{5}{*}{ Drug treatment } & & & Fold reduction of $\mathrm{IC}_{50}$ \\
\hline & & $\begin{array}{l}\text { qRT- } \\
\text { PCR }\end{array}$ & \\
\hline & $\begin{array}{l}\text { Chloroquine + Niclosamide } \\
0.0045 \mu \mathrm{M}\end{array}$ & 0.193 & 4.61 \\
\hline & $\begin{array}{l}\text { Chloroquine + Niclosamide } \\
0.0225 \mu \mathrm{M}\end{array}$ & 0.249 & 3.57 \\
\hline & $\begin{array}{l}\text { Chloroquine + Niclosamide } \\
0.01125 \mu \mathrm{M}\end{array}$ & 0.531 & 1.68 \\
\hline \multirow{10}{*}{$\begin{array}{l}\text { Ivermectin- } \\
\text { Chloroquine }\end{array}$} & Ivermectin & 1.27 & \\
\hline & $\begin{array}{l}\text { Ivermectin + Chloroquine } 1.7 \\
\mu \mathrm{M}\end{array}$ & 0.023 & 55.22 \\
\hline & $\begin{array}{l}\text { Ivermectin + Chloroquine } 0.85 \\
\mu \mathrm{M}\end{array}$ & 0.122 & 10.41 \\
\hline & $\begin{array}{l}\text { Ivermectin + Chloroquine } 0.425 \\
\mu \mathrm{M}\end{array}$ & 0.515 & 2.47 \\
\hline & $\begin{array}{l}\text { Ivermectin + Chloroquine } \\
0.2125 \mu \mathrm{M}\end{array}$ & 0.821 & 1.55 \\
\hline & Chloroquine & 0.89 & \\
\hline & $\begin{array}{l}\text { Chloroquine + Ivermectin } 2.4 \\
\mu \mathrm{M}\end{array}$ & 0.014 & 63.57 \\
\hline & $\begin{array}{l}\text { Chloroquine + Ivermectin } 1.2 \\
\mu \mathrm{M}\end{array}$ & 0.221 & 4.03 \\
\hline & $\begin{array}{l}\text { Chloroquine + Ivermectin } 0.6 \\
\mu \mathrm{M}\end{array}$ & 0.315 & 2.83 \\
\hline & $\begin{array}{l}\text { Chloroquine + Ivermectin } 0.3 \\
\mu \mathrm{M}\end{array}$ & 0.514 & 1.73 \\
\hline
\end{tabular}

\section{Niclosamide-chloroquine Combination}

By using the same approach, it was found that the presence of Chloroquine induced a shift in Niclosamide dose-response curve, with $14.333,4.778,3.308$ and 1.483 -fold reduction of Niclosamide $\mathrm{IC}_{50}$ value in the presence of $1.7,0.85,0.425$, and $0.2125 \mu \mathrm{M}$ Chloroquine, respectively (Fig. 3A, Table 2). A similar trend was observed for the Chloroquine dose-response curve in the presence of Niclosamide, with $31.78,4.61,3.57$ and 1.68 -fold reduction of Chloroquine $\mathrm{IC}_{50}$ value in the presence of $0.09,0.045,0.0225$ and $0.01125 \mu \mathrm{M}$ Niclosamide, respectively (Fig. 3B, Table 2). The dose-response matrix shows increasing 
inhibitory effect of the combination with higher concentrations of Niclosamide and Chloroquine (Fig. 3C). The synergy map shows positive synergy scores at high concentrations of both drugs, while the lower concentrations gave zero and negative synergy scores with a peak positive score of 18.57 , indicating a synergistic effect. (Fig. 3D). As most parts of the surface had Loewe synergy scores between -10 and 10, except for the highest concentrations of both drugs, with a mean score of 0.073 , it suggests an additive effect between Niclosamide and Chloroquine. Additionally, the synergy scores calculated using ZIP and Bliss independence reference models gave the values of 3.86 and 3.67, respectively, which similarly indicated the additive effect. The HSA model resulted in the synergy score of 11.41, which accounted for the small level in synergistic effect. No significant cytotoxicity in all 16 pairwise combinations (Fig. 3A, B).

\section{Ivermectin-chloroquine Combination}

The results showed that the presence of Chloroquine induced a shift in Ivermectin dose-response curve, with $55.22,10.41,2.47,1.55$-fold reduction of Ivermectin $\mathrm{IC}_{50}$ value in the presence of $1.7,0.85,0.425$, and $0.2125 \mu \mathrm{M}$ Chloroquine, respectively (Fig. 4A, Table 2). Similarly, the presence of Ivermectin also induced a shift in Chloroquine dose-response curve, with 63.57, 4.03, 2.83 and 1.73-fold reduction of Chloroquine $\mathrm{IC}_{50}$ value in the presence of $2.4,1.2,0.6$ and $0.3 \mu \mathrm{M}$ Ivermectin, respectively (Fig. 4B, Table 2). The dose-response matrix shows increasing inhibitory effect with higher concentrations of Ivermectin and Chloroquine (Fig. 4C). Most parts of the synergy surface show negative synergy scores except for a small positive area with a peak positive score of 7.43 at the highest concentration of Chloroquine (Fig. 4D). The peak negative score of the surface is -8.16. As all of the surface had Loewe synergy scores between -10 and 10 with a mean score of -1.812 , it suggests an additive effect between Ivermectin and Chloroquine. Moreover, both ZIP, Bliss independence and HSA reference models showed the synergy scores of $1.97,1.98$ and 9.63 which indicated the additive effect. No significant cytotoxicity in all 16 pairwise combinations (Fig. 4A, B).

\section{Evaluation of single drug treatment against SARS-CoV-2 in Calu-3 cells}

The best antiviral activity and calculated synergy scores demonstrated in the treatment with NiclosamideIvermectin combination in Vero E6 cells. Therefore, this two-drug combination was selected for the further evaluation in the human lung cancer cell line, Calu-3. The antiviral activities of single Niclosamide and Ivermectin treatments were assessed in Calu-3 cells (Fig. 5). The $\mathrm{IC}_{50}$ values of both drugs were $0.2 \mu \mathrm{M}$ in Calu-3 cells. The $\mathrm{CC}_{50}$ values of Niclosamide and Ivermectin were $5.62 \mu \mathrm{M}$ and $3.10 \mu \mathrm{M}$, respectively. The SI values of Niclosamide and Ivermectin were 28.1 and 15.5, respectively.

\section{Evaluation of Niclosamide-Ivermectin combination treatment against SARS-CoV-2 in Calu-3 cells}

The strong shifts were observed in the dose-response curves of Niclosamide combined with 0.4 and 0.2 $\mu \mathrm{M}$ Ivermectin (Fig. 6A, Table 3). The pairwise combinations of four different concentrations of Niclosamide with 0.4 and $0.2 \mu \mathrm{M}$ Ivermectin resulted in a similar percent inhibition, thus, it was unable to 
calculate accurate $\mathrm{IC}_{50}$ values with the least curve fit. The presence of 0.1 and $0.05 \mu \mathrm{M}$ Ivermectin also induced a shift in the dose-response curve of Niclosamide, in a similar level of 2.38 and 2.33-fold reduction of Niclosamide IC $\mathrm{C}_{50}$ values, respectively (Fig. 6A, Table 3). In a similar way, the presence of Niclosamide induced a shift in Ivermectin dose-response curve with $8.69,4.88,3.64$, and 2.41-fold reduction of Ivermectin $\mathrm{IC}_{50}$ value in the presence of $0.4,0.2,0.1$ and $0.05 \mu \mathrm{M}$ Niclosamide, respectively (Fig. 6B, Table 3). The dose-response matrix shows the increasing antiviral activity compared to the single drug treatments (Fig. 6C). The combination synergy analysis showed the mean Loewe synergy score of 2.897, which accounted for the additive effect between Niclosamide and Ivermectin in Calu-3 cells (Fig. 6D). Additionally, the peak Loewe synergy score was 13.19. The synergy score obtained from ZIP and Bliss independence reference models were 0.886 and 0.954 , respectively, which also accounted for the additive effect. The synergy score calculated using HSA models was 10.795, which indicated a small synergistic effect between Niclosamide and Ivermectin. All 16 pairwise combinations showed no significant cytotoxicity (Fig. 6A, B)

Table 3

Evaluation of Niclosamide-lvermectin combination treatments against SARS-CoV-2 in Calu-3 cells

\begin{tabular}{|c|c|c|c|}
\hline Drug treatment & & $\begin{array}{l}\mathrm{IC}_{50} \\
(\mu \mathrm{M})\end{array}$ & $\begin{array}{l}\text { Fold reduction of } \mathrm{IC}_{50} \\
\text { (single/combined) }\end{array}$ \\
\hline \multirow{10}{*}{$\begin{array}{l}\text { Favipiravir- } \\
\text { Ivermectin }\end{array}$} & Favipiravir & 0.20 & \\
\hline & $\begin{array}{l}\text { Niclosamide + Ivermectin } 0.4 \\
\mu \mathrm{M}\end{array}$ & ND & ND \\
\hline & $\begin{array}{l}\text { Niclosamide + Ivermectin } 0.2 \\
\mu \mathrm{M}\end{array}$ & ND & ND \\
\hline & $\begin{array}{l}\text { Niclosamide + Ivermectin } 0.1 \\
\mu \mathrm{M}\end{array}$ & 0.084 & 2.38 \\
\hline & $\begin{array}{l}\text { Niclosamide + Ivermectin } 0.05 \\
\mu \mathrm{M}\end{array}$ & 0.086 & 2.33 \\
\hline & Ivermectin & 0.20 & \\
\hline & $\begin{array}{l}\text { Ivermectin + Niclosamide } 0.4 \\
\mu \mathrm{M}\end{array}$ & 0.023 & 8.69 \\
\hline & $\begin{array}{l}\text { Ivermectin + Niclosamide } 0.2 \\
\mu \mathrm{M}\end{array}$ & 0.041 & 4.88 \\
\hline & $\begin{array}{l}\text { Ivermectin + Niclosamide } 0.1 \\
\mu \mathrm{M}\end{array}$ & 0.055 & 3.64 \\
\hline & $\begin{array}{l}\text { Ivermectin + Niclosamide } 0.05 \\
\mu \mathrm{M}\end{array}$ & 0.083 & 2.41 \\
\hline
\end{tabular}




\section{Discussion}

Our study shows that the repurposed anti-parasitic drugs, Niclosamide, Ivermectin and Chloroquine possess high in vitro activity against SARS-CoV-2 as the $\mathrm{IC}_{50}$ values are in the low micromolar range. These results on the $\mathrm{IC}_{50}$ against SARS-CoV-2 of these single drugs are in agreement with previous studies $[9,20,28,30]$.

Previous in vitro studies suggested that Ivermectin inhibits host importin alpha/beta-1 nuclear transport proteins, thus preventing the viruses from suppressing the host antiviral response [47]. Recently, it was found that Ivermectin may interfere with the attachment of SARS-CoV-2 spike protein to the ACE2 receptor on human cell membrane [23]. Several studies also reported antiviral activity of Ivermectin on other viruses such as Zika virus [5], Dengue virus [42] and Human immunodeficiency virus type 1(HIV-1) [41]. And with its board spectrum antiviral activity, Ivermectin is thought to act on host cells for its antiviral activity.

Niclosamide showed broad antiviral activity against a wide range of viruses such as SARS-CoV [43-45], MERS-CoV [15], Zika virus [46], HCV [36], Ebola virus [27] and HIV-1 [29]. Several evidences found in other viruses suggested the plausible mechanisms of Niclosamide in SARS-CoV-2 inhibition by blocking of viral entry via altering endosomal $\mathrm{pH}$ and the prevention of autophagy that lead to the inhibition of virus replication $[15,21,37]$. Although Niclosamide was originally thought to act on parasitic worms in the gut lumen and is barely absorbed to the blood stream, it was tested for various systemic repurposed treatments, and a maximal plasma concentration ranged from 35.7 to $182 \mathrm{ng} \mathrm{ml}^{-1}$ (corresponding to 0.11-0.56 $\mu \mathrm{M}$ ) was observed in a pharmacokinetic study $[3,8,24,34]$. This level exceeds the in vitro Niclosamide $\mathrm{IC}_{50}$ against SARS-CoV-2, especially when used in the tested combinations.

Chloroquine inhibits a broad range of viruses by blocking viral entry via inhibition of endosomal acidification [33]. It was recently shown that Chloroquine could not inhibit SARS-CoV-2 in human lung cells because of the expression of TMPRSS2 [16]. This may at least partially explain the lack of clinical efficacy of this drug. Despite these in vitro anti-SARS-CoV-2 activities, clinical application of these drugs to COVID-19 treatment has not yet been successful. While some clinical trials of Ivermectin on COVID-19 treatment have shown promising results $[1,6,11,12,17,25,40]$, clinical trials for Chloroquine mostly showed negative results [35] and there have been little clinical data on Niclosamide. The lack of obvious clinical efficacy suggests that either these in vitro activities could not take effect in vivo or the activities may not be sufficiently potent. An obvious strategy to enhance the potency is drug combination. While combining direct acting antivirals with different targets almost always results in additive or synergistic effect, combining drugs that act on host machineries does not always cause a synergistic effect and can even result in an antagonistic effect $[7,30]$. Selecting proper drug combinations with synergistic effect is therefore crucial for development of efficacious regimens. Our data may be useful in guiding the design of clinical trials that may generate a badly needed efficacious regimen for COVID-19 treatment and prevention. 
In conclusion, our study demonstrated the benefit of combining Ivermectin, Niclosamide and Chloroquine on their anti-SAR-CoV-2 activities. Among the combinations, Ivermectin and Niclosamide showed the best synergistic profile. This combination should be further tested in clinical trials.

\section{Declarations}

\section{Acknowledgement}

This study was supported by the Chair Professor Program (P-20-52262), The National Science and Technology Development Agency (NSTDA), Thailand. This study was also partly supported by Faculty of Medicine Siriraj Hospital, Mahidol University, Thailand.

\section{Author Contributions}

KJ (Methodology, Formal Analysis, Investigation, Writing - Original Draft Preparation), CB (Methodology, Formal Analysis, Investigation), SM (Methodology, Investigation), NP (Methodology, Investigation), SB (Resource), AT (Validation, Methodology), PA (Validation, Methodology), P. Auewarakul (Conceptualization, Supervision, Funding, Writing - Review and Editing). All authors have read and agreed to the published version of the manuscript.

\section{Conflicts of interest}

The authors declare that they have no conflicts of interest.

\section{Biosafety}

This study was approved by Mahidol University Biosafety Committee (approval no. MU 2020-008).

\section{References}

1. Ahmed S, Karim MM, Ross AG, Hossain MS, Clemens JD, Sumiya MK, Phru CS, Rahman M, Zaman K, Somani J, Yasmin R, Hasnat MA, Kabir A, Aziz AB, Khan WA (2021) A five-day course of ivermectin for the treatment of COVID-19 may reduce the duration of illness. Int J Infect Dis 103:214-216

2. American Library Association (2021) COVID-19 Coronavirus pandemic Worldometer

3. Andrews P, Thyssen J, Lorke D (1982) The biology and toxicology of molluscicides, Bayluscide. Pharmacol Ther 19:245-295

4. Arshad U, Pertinez H, Box H, Tatham L, Rajoli RKR, Curley P, Neary M, Sharp J, Liptrott NJ, Valentijn A, David C, Rannard SP, O'Neill PM, Aljayyoussi G, Pennington SH, Ward SA, Hill A, Back DJ, Khoo SH, Bray PG, Biagini GA, Owen A (2020) Prioritization of anti-SARS-CoV-2 drug repurposing opportunities based on plasma and target site concentrations derived from their established human pharmacokinetics. Clin Pharmacol Ther 108:775-790 
5. Barrows NJ, Campos RK, Powell ST, Prasanth KR, Schott-Lerner G, Soto-Acosta R, Galarza-Muñoz G, McGrath EL, Urrabaz-Garza R, Gao J, Wu P, Menon R, Saade G, Fernandez-Salas I, Rossi SL, Vasilakis N, Routh A, Bradrick SS, Garcia-Blanco MA (2016) A screen of FDA-approved drugs for inhibitors of Zika virus infection. Cell Host Microbe 20:259-270

6. Behera P, Patro BK, Singh AK, Chandanshive PD, S. R R, Pradhan SK, Pentapati SSK, Batmanabane G, Mohapatra PR, Padhy BM, Bal SK, Singh SR, Mohanty RR (2021) Role of ivermectin in the prevention of SARS-CoV-2 infection among healthcare workers in India: A matched case-control study. PLOS ONE 16:e0247163

7. Bobrowski T, Chen L, Eastman RT, Itkin Z, Shinn P, Chen CZ, Guo H, Zheng W, Michael S, Simeonov A, Hall MD, Zakharov AV, Muratov EN (2021) Synergistic and antagonistic drug combinations against SARS-CoV-2. Mol Ther 29:873-885

8. Burock S, Daum S, Keilholz U, Neumann K, Walther W, Stein U (2018) Phase II trial to investigate the safety and efficacy of orally applied niclosamide in patients with metachronous or sychronous metastases of a colorectal cancer progressing after therapy: the NIKOLO trial. BMC Cancer 18:297

9. Caly L, Druce JD, Catton MG, Jans DA, Wagstaff KM (2020) The FDA-approved drug ivermectin inhibits the replication of SARS-CoV-2 in vitro. Antiviral Res 178:104787

10. Camprubí D, Almuedo-Riera A, Martí-Soler H, Soriano A, Hurtado JC, Subirà C, Grau-Pujol B, Krolewiecki A, Muñoz J (2020) Lack of efficacy of standard doses of ivermectin in severe COVID-19 patients. PLOS ONE 15:e0242184

11. Chaccour C, Ruiz-Castillo P, Richardson M-A, Moncunill G, Casellas A, Carmona-Torre F, Giráldez M, Mota JS, Yuste JR, Azanza JR, Fernández M, Reina G, Dobaño C, Brew J, Sadaba B, Hammann F, Rabinovich R (2020) The SARS-CoV-2 Ivermectin Navarra-ISGlobal Trial (SAINT) to evaluate the potential of ivermectin to reduce COVID-19 transmission in low risk, non-severe COVID-19 patients in the first 48 hours after symptoms onset: A structured summary of a study protocol for a randomized control pilot trial. Trials 21:498

12. Chaccour C, Casellas A, Blanco-Di Matteo A, Pineda I, Fernandez-Montero A, Ruiz-Castillo P, Richardson MA, Rodríguez-Mateos M, Jordán-Iborra C, Brew J, Carmona-Torre F, Giráldez M, Laso E, Gabaldón-Figueira JC, Dobaño C, Moncunill G, Yuste JR, Del Pozo JL, Rabinovich NR, Schöning V, Hammann F, Reina G, Sadaba B, Fernández-Alonso M (2021) The effect of early treatment with ivermectin on viral load, symptoms and humoral response in patients with non-severe COVID-19: A pilot, double-blind, placebo-controlled, randomized clinical trial. EClinicalMedicine 32:100720

13. Elalfy $H$, Besheer T, El-Mesery A, El-Gilany A-H, Abd Elazez MS, Alhawarey A, Alegezy M, Elhadidy T, Hewidy AA, Zaghloul H, Neamatallah MAM, Raafat D, El-Emshaty WM, Abo El Kheir NY, El-Bendary M (2021) Effect of a combination of nitazoxanide, ribavirin, and ivermectin plus zinc supplement (MANS.NRIZ study) on the clearance of mild COVID-19. n/a

14. Ganguly D, Rottet S, Yee S, Hee W, Smith A, Khin N, Millar A, Fahrer A (2020) SYBR green one-step qRT-PCR for the detection of SARS-CoV-2 RNA in saliva.2020.2005.2029.109702 
15. Gassen NC, Niemeyer D, Muth D, Corman VM, Martinelli S, Gassen A, Hafner K, Papies J, Mösbauer K, Zellner A, Zannas AS, Herrmann A, Holsboer F, Brack-Werner R, Boshart M, Müller-Myhsok B, Drosten C, Müller MA, Rein T (2019) SKP2 attenuates autophagy through Beclin1-ubiquitination and its inhibition reduces MERS-Coronavirus infection. Nat Commun 10:5770

16. Hoffmann M, Mösbauer K, Hofmann-Winkler H, Kaul A, Kleine-Weber H, Krüger N, Gassen NC, Müller MA, Drosten C, Pöhlmann S (2020) Chloroquine does not inhibit infection of human lung cells with SARS-CoV-2. Nature 585:588-590

17. Hosseini FS, Malektojari A, Ghazizadeh S, Hassaniazad M, Davoodian P, Dadvand H, Nikpoor AR, Nikoofal-Sahlabadi S, Kahoori S, Sepandi M, Hassanipour S, Fathalipour M (2021) The efficacy and safety of Ivermectin in patients with mild and moderate COVID-19: A structured summary of a study protocol for a randomized controlled trial. Trials 22:4

18. lanevski A, Giri AK, Aittokallio T (2020) SynergyFinder 2.0: visual analytics of multi-drug combination synergies. Nucleic Acids Res 48:W488-W493

19. Jans DA, Wagstaff KM (2021) The broad spectrum host-directed agent ivermectin as an antiviral for SARS-CoV-2 ? Biochem Biophys Res Commun 538:163-172

20. Jeon S, Ko M, Lee J, Choi I, Byun SY, Park S, Shum D, Kim S (2020) Identification of antiviral drug candidates against SARS-CoV-2 from FDA-approved drugs. Antimicrob Agents Chemother 64:e00819-00820

21. Jurgeit A, McDowell R, Moese S, Meldrum E, Schwendener R, Greber UF (2012) Niclosamide is a proton carrier and targets acidic endosomes with broad antiviral effects. PLoS Pathog 8:e1002976

22. Kanjanasirirat P, Suksatu A, Manopwisedjaroen S, Munyoo B, Tuchinda P, Jearawuttanakul K, Seemakhan S, Charoensutthivarakul S, Wongtrakoongate P, Rangkasenee N, Pitiporn S, Waranuch N, Chabang N, Khemawoot P, Sa-ngiamsuntorn K, Pewkliang Y, Thongsri P, Chutipongtanate S, Hongeng S, Borwornpinyo S, Thitithanyanont A (2020) High-content screening of Thai medicinal plants reveals Boesenbergia rotunda extract and its component Panduratin A as anti-SARS-CoV-2 agents. Sci Rep 10:19963

23. Lehrer S, Rheinstein PH (2020) Ivermectin Docks to the SARS-CoV-2 Spike Receptor-binding Domain Attached to ACE2. In Vivo 34:3023-3026

24. Li Y, Li PK, Roberts MJ, Arend RC, Samant RS, Buchsbaum DJ (2014) Multi-targeted therapy of cancer by niclosamide: A new application for an old drug. Cancer Lett 349:8-14

25. Lima-Morales R, Méndez-Hernández P, Flores YN, Osorno-Romero P, Cuecuecha-Rugerio E, NavaZamora A, Hernández-Galdamez DR, Romo-Dueñas DK, Salmerón J (2021) Effectiveness of a multidrug therapy consisting of ivermectin, azithromycin, montelukast and acetylsalicylic acid to prevent hospitalization and death among ambulatory COVID-19 cases in Tlaxcala, Mexico. Int J Infect Dis

26. Loewe $S$ (1953) The problem of synergism and antagonism of combined drugs. Arzneimittelforschung 3:285-290 
27. Madrid PB, Panchal RG, Warren TK, Shurtleff AC, Endsley AN, Green CE, Kolokoltsov A, Davey R, Manger ID, Gilfillan L, Bavari S, Tanga MJ (2015) Evaluation of Ebola virus inhibitors for drug repurposing. ACS Infect Dis 1:317-326

28. Mostafa A, Kandeil A, Y AMME, Kutkat O, Moatasim Y, Rashad AA, Shehata M, Gomaa MR, Mahrous N, Mahmoud SH, GabAllah M, Abbas H, Taweel AE, Kayed AE, Kamel MN, Sayes ME, Mahmoud DB, El-Shesheny R, Kayali G, Ali MA (2020) FDA-approved drugs with potent in vitro antiviral activity against severe acute respiratory syndrome coronavirus 2 . Pharm $\mathrm{J} 13$

29. Niyomdecha N, Suptawiwat O, Boonarkart C, Jitobaom K, Auewarakul P (2020) Inhibition of human immunodeficiency virus type 1 by niclosamide through mTORC1 inhibition. Heliyon 6:e04050

30. Pizzorno A, Padey B, Dubois J, Julien T, Traversier A, Dulière V, Brun P, Lina B, Rosa-Calatrava M, Terrier $O$ (2020) In vitro evaluation of antiviral activity of single and combined repurposable drugs against SARS-CoV-2. Antiviral Res 181:104878

31. Reed LJ, Muench H (1938) A simple method of estimating fifty percent enpoint Am J Epidemiol 27:493-497

32. Remme J, De Sole G, Dadzie KY, Alley ES, Baker RH, Habbema JD, Plaisier AP, van Oortmarssen GJ, Samba EM (1990) Large scale ivermectin distribution and its epidemiological consequences. Acta Leiden 59:177-191

33. Savarino A, Boelaert JR, Cassone A, Majori G, Cauda R (2003) Effects of chloroquine on viral infections: an old drug against today's diseases? Lancet Infect Dis 3:722-727

34. Schweizer MT, Haugk K, McKiernan JS, Gulati R, Cheng HH, Maes JL, Dumpit RF, Nelson PS, Montgomery B, McCune JS, Plymate SR, Yu EY (2018) A phase I study of niclosamide in combination with enzalutamide in men with castration-resistant prostate cancer. PLOS ONE 13:e0198389-e0198389

35. Singh B, Ryan H, Kredo T, Chaplin M, Fletcher T (2021) Chloroquine or hydroxychloroquine for prevention and treatment of COVID-19. Cochrane Database Syst Rev

36. Stachulski AV, Pidathala C, Row EC, Sharma R, Berry NG, Lawrenson AS, Moores SL, Iqbal M, Bentley J, Allman SA, Edwards G, Helm A, Hellier J, Korba BE, Semple JE, Rossignol J-F (2011) Thiazolides as novel antiviral agents. 2. inhibition of hepatitis $C$ virus replication. J Med Chem 54:8670-8680

37. Thomson G (2020) COVID-19: Social distancing, ACE 2 receptors, protease inhibitors and beyond? Int $\mathrm{J}$ Clin Pract 74:e13503

38. US FDA (2020) Coronavirus (COVID-19) Update: FDA Authorizes Drug Combination for Treatment of COVID-19.

39. US FDA (2020) FDA Approves First Treatment for COVID-19.

40. Vallejos J, Zoni R, Bangher M, Villamandos S, Bobadilla A, Plano F, Campias C, Chaparro Campias E, Achinelli F, Guglielmone HA, Ojeda J, Medina F, Farizano Salazar D, Andino G, Ruiz Diaz NE, Kawerin P, Meza E, Dellamea S, Aquino A, Flores V, Martemucci CN, Vernengo MM, Martinez SM, Segovia JE, Aguirre MG (2020) Ivermectin to prevent hospitalizations in patients with COVID-19 (IVERCORCOVID19): a structured summary of a study protocol for a randomized controlled trial. Trials 21:965 
41. Wagstaff KM, Rawlinson SM, Hearps AC, Jans DA (2011) An AlphaScreen®-based assay for highthroughput screening for specific inhibitors of nuclear import. J Biomol Screen 16:192-200

42. Wagstaff KM, Sivakumaran $H$, Heaton SM, Harrich D, Jans DA (2012) Ivermectin is a specific inhibitor of importin $\alpha / \beta$-mediated nuclear import able to inhibit replication of HIV- 1 and dengue virus. Biochem J 443:851-856

43. Wen C-C, Kuo Y-H, Jan J-T, Liang P-H, Wang S-Y, Liu H-G, Lee C-K, Chang S-T, Kuo C-J, Lee S-S, Hou CC, Hsiao P-W, Chien S-C, Shyur L-F, Yang N-S (2007) Specific plant terpenoids and lignoids possess potent antiviral activities against severe acute respiratory syndrome coronavirus. J Med Chem 50:4087-4095

44. Wu C-J, Jan J-T, Chen C-M, Hsieh H-P, Hwang D-R, Liu H-W, Liu C-Y, Huang H-W, Chen S-C, Hong C-F, Lin R-K, Chao Y-S, Hsu JTA (2004) Inhibition of severe acute respiratory syndrome coronavirus replication by Niclosamide. Antimicrob Agents Chemother 48:2693-2696

45. Xu J, Shi P-Y, Li H, Zhou J (2020) Broad Spectrum Antiviral Agent Niclosamide and Its Therapeutic Potential. ACS Infect Dis 6:909-915

46. Xu M, Lee EM, Wen Z, Cheng Y, Huang WK, Qian X, Tcw J, Kouznetsova J, Ogden SC, Hammack C, Jacob F, Nguyen HN, Itkin M, Hanna C, Shinn P, Allen C, Michael SG, Simeonov A, Huang W, Christian KM, Goate A, Brennand KJ, Huang R, Xia M, Ming GL, Zheng W, Song H, Tang H (2016) Identification of small-molecule inhibitors of Zika virus infection and induced neural cell death via a drug repurposing screen. Nat Med 22:1101-1107

47. Yang SNY, Atkinson SC, Wang C, Lee A, Bogoyevitch MA, Borg NA, Jans DA (2020) The broad spectrum antiviral ivermectin targets the host nuclear transport importin $\alpha / \beta 1$ heterodimer. Antivir Res 177:104760

\section{Figures}
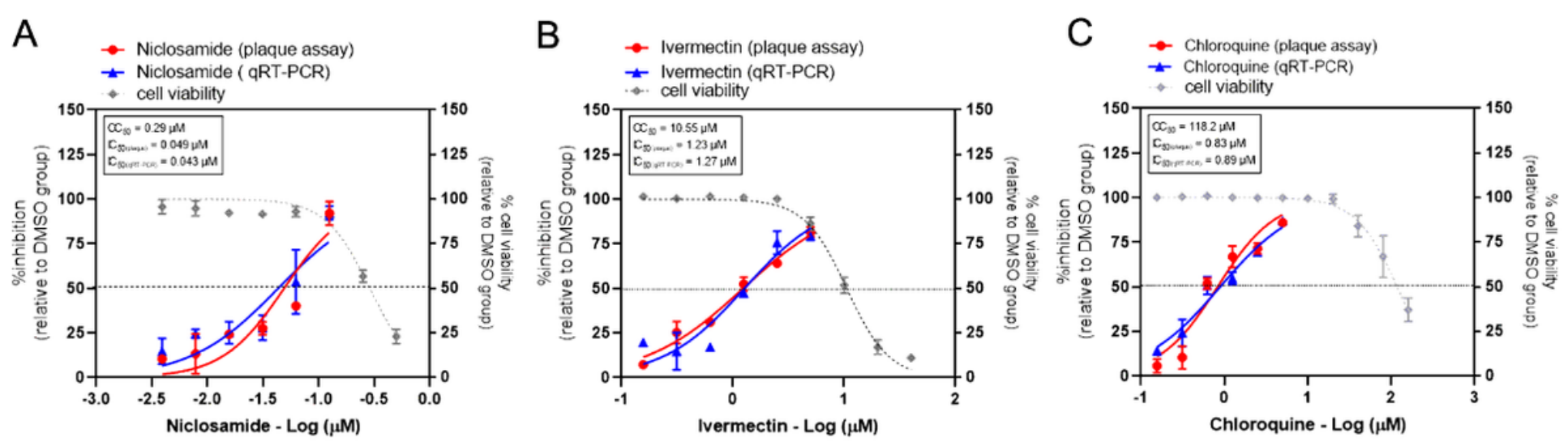

Figure 1

Evaluation of antiviral activity of drug candidates against SARS-CoV-2 in vitro The dose-response curves of a single drug treatment against SARS-CoV-2 were shown; (A) Niclosamide, (B) Ivermectin, and (C) 
Chloroquine. Vero E6 cells were treated with twofold serial dilutions of drug for one hour and infected with SARS-CoV-2 at m.o.i. of 0.01. After removing of viruses, the cells were maintained in the medium containing serial dilutions of the single drugs or $0.5 \% \mathrm{DMSO}$ for two days. The virus supernatants were collected for titration using the plaque assay and one step-qRT-PCR. The dose-response curves were expressed as the percent inhibition in relative to the infected-DMSO-treated cell control. The effect of drug treatment on the cell viability was determined using MTT assay and is expressed in relative to the DMSOtreated cell control. The experiments were repeated at least three times, and data are shown as mean \pm SD. 
A

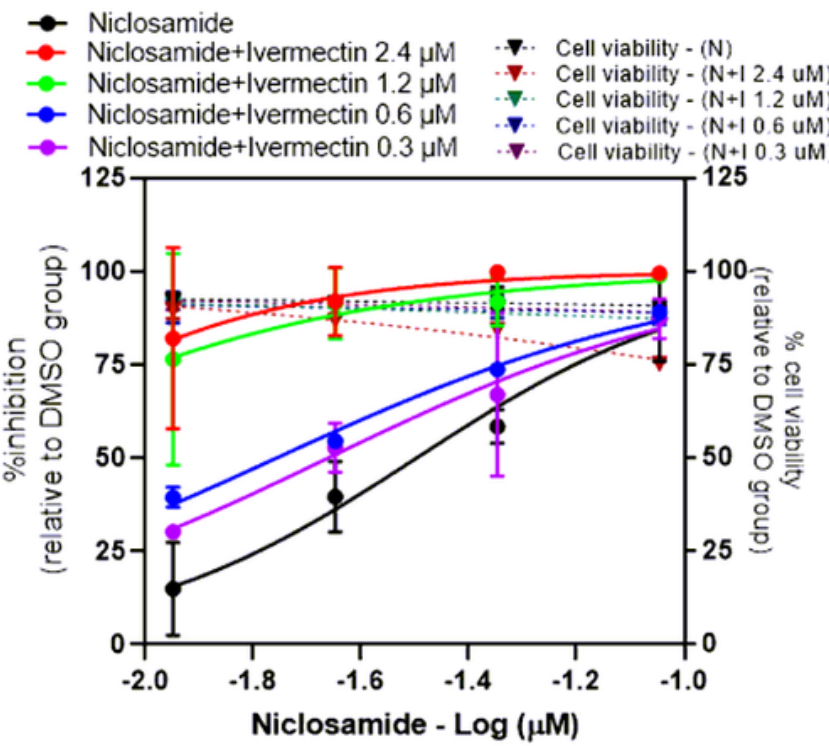

C

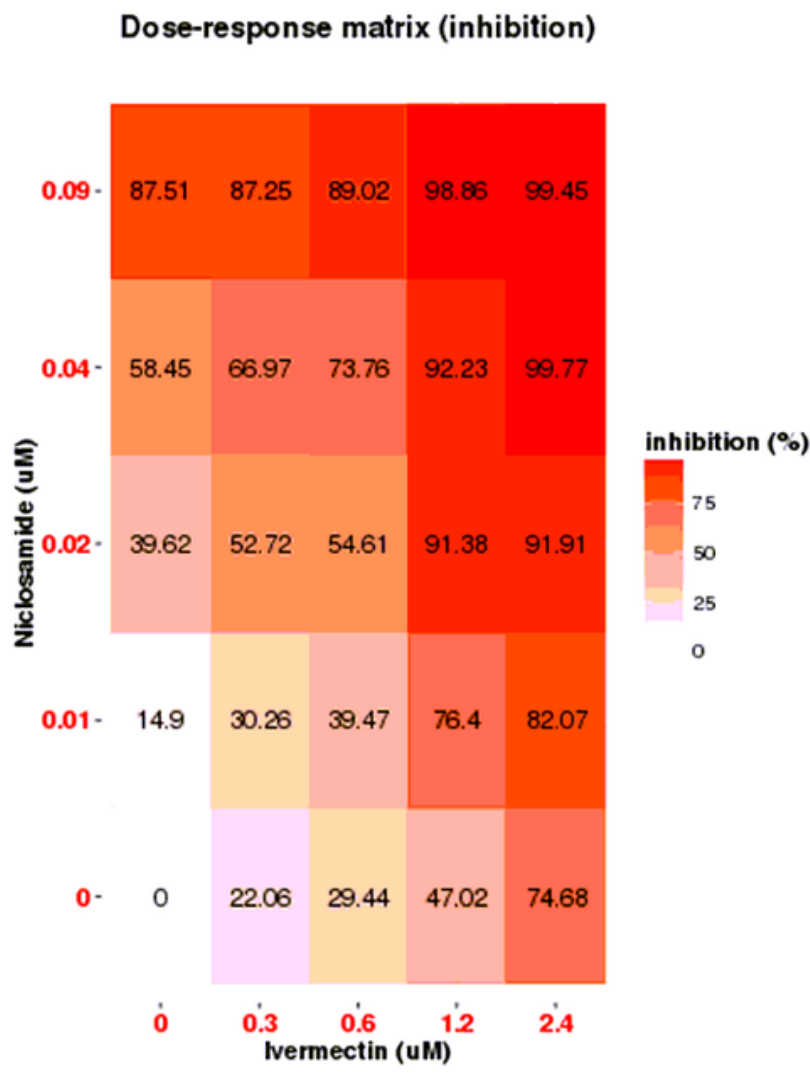

B

$\rightarrow$ Ivermectin

- Ivermectin+Niclosamide $0.09 \mu \mathrm{M} \quad-\nabla \cdot$ cell viability (I)

- Ivermectin+Niclosamide $0.045 \mu \mathrm{M} \quad-\nabla \cdot$ cell viablity $-(1+\mathrm{N} 0.09 \mu \mathrm{M})$

$\rightarrow$ Ivermectin+Niclosamide $0.0225 \mu \mathrm{M}-\boldsymbol{\nabla} \cdot$. cell viablity $-(\mathrm{I}+\mathrm{N} 0.0045 \mu \mathrm{M})$

- Ivermectin+Niclosamide $0.01125 \mu \mathrm{M}-\boldsymbol{\nabla} \cdot$ cell viablity $-(1+\mathrm{N} 0.0225 \mu \mathrm{M})$

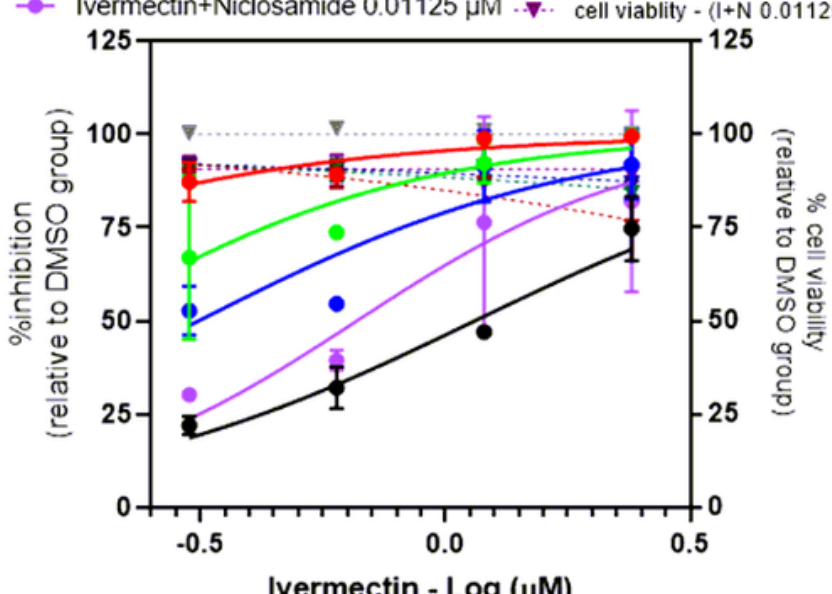

Ivermectin - $\log (\mu \mathrm{M})$

D
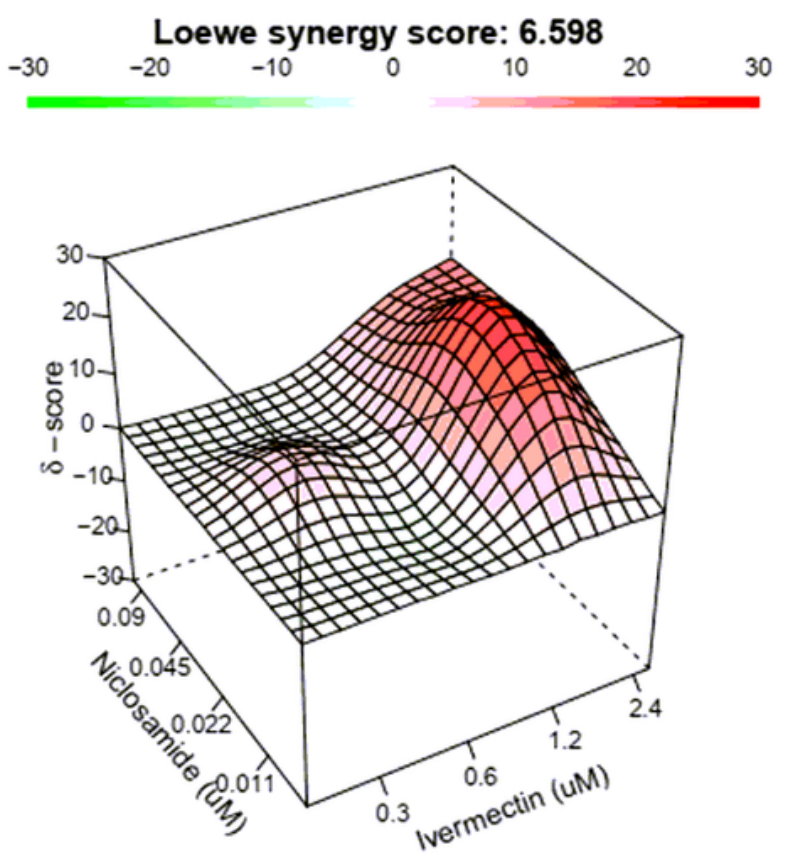

\section{Figure 2}

2Niclosamide-Ivermectin combination treatments against SARS-CoV-2 in Vero E6 cells. Vero E6 cells were treated for one hour with twofold serial dilutions of Niclosamide in the presence of different fixed concentrations of Ivermectin (A) or alternatively, serial dilutions of Ivermectin in the presence of different fixed concentrations of Niclosamide (B). Then the cells were infected with SARS-CoV-2 at m.o.i. 0.01. After removing the virus inoculum, the cells were further maintained in the medium containing drugs for 2 
days. The viral RNA was determined using one-step qRT-PCR. The SynergyFinder was used to calculate the synergy score of two-drug combinations from different 16 pairwise combinations. The dose-response matrix (C) and the synergy map of two-drug combinations treatment (D) were shown. The interaction landscape between two drugs was calculated using Loewe model. Areas with synergy score less than -10 : the interaction between two drugs is likely to be antagonistic; from -10 to 10: the interaction between two drugs is likely to be additive; larger than 10: the interaction between two drugs is likely to be synergistic. The experiments were repeated at least three times, and data are shown as mean \pm SD.

A

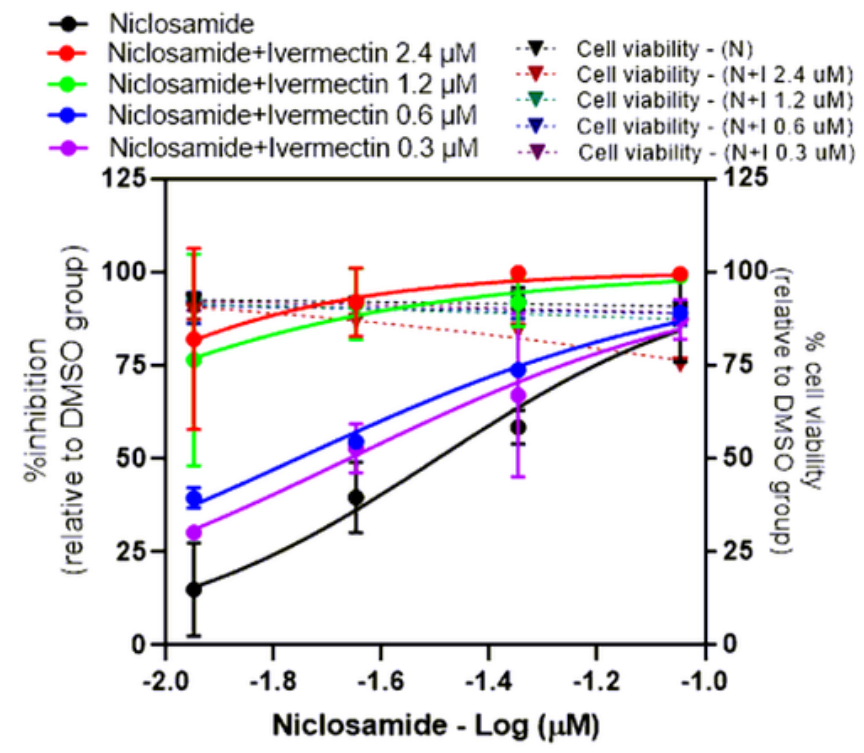

C

Dose-response matrix (inhibition)

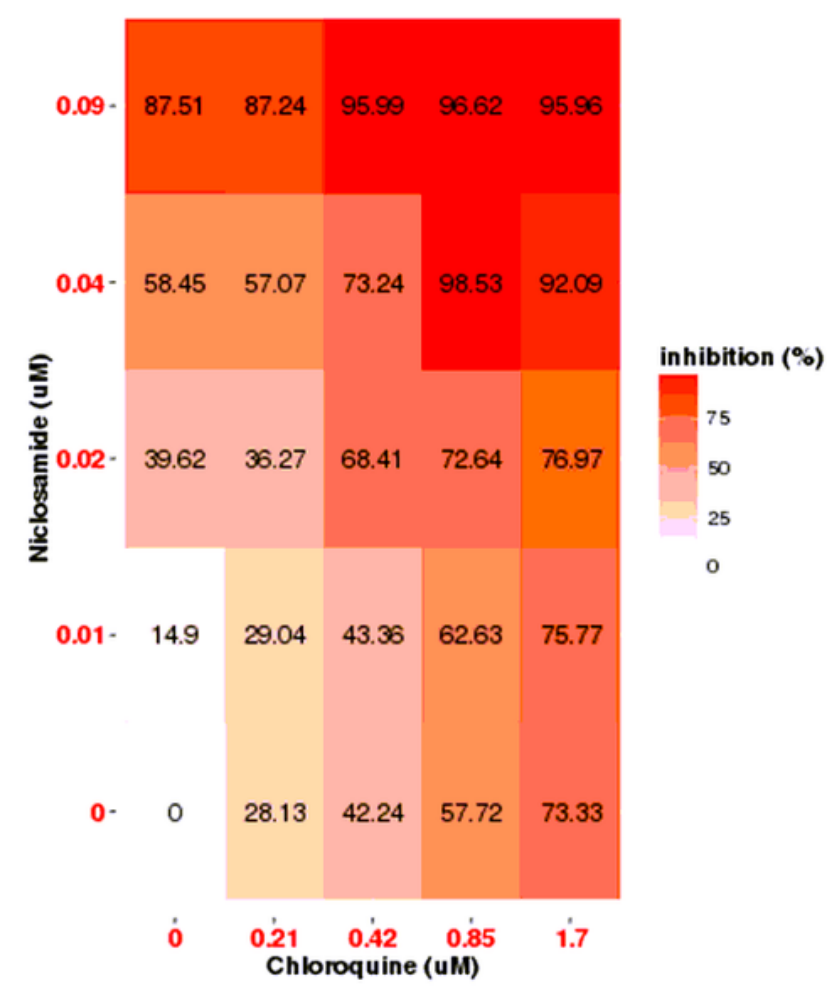

B

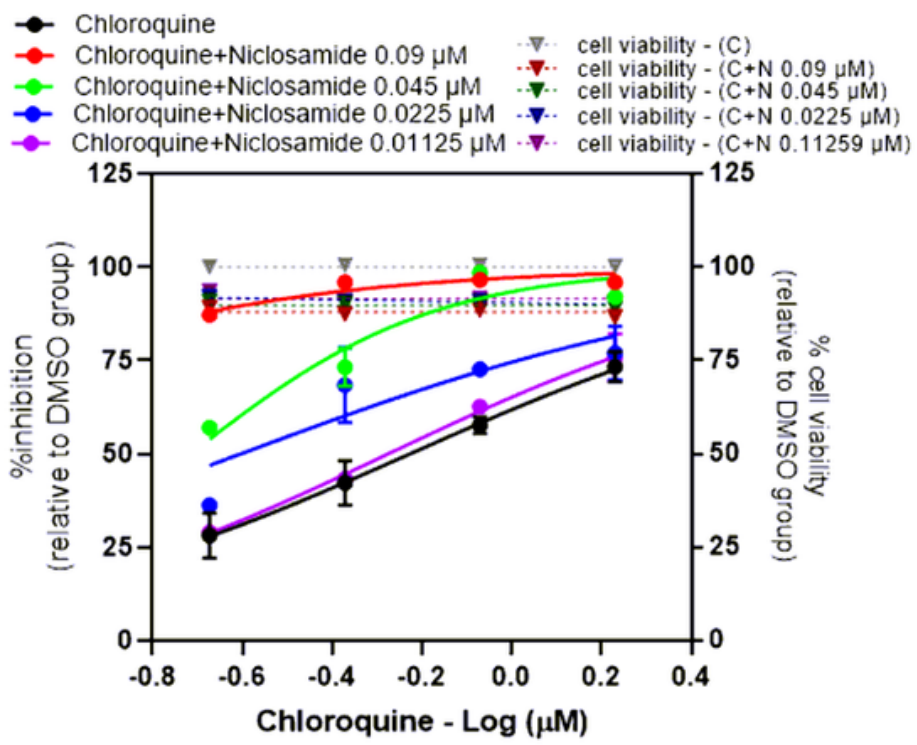

D
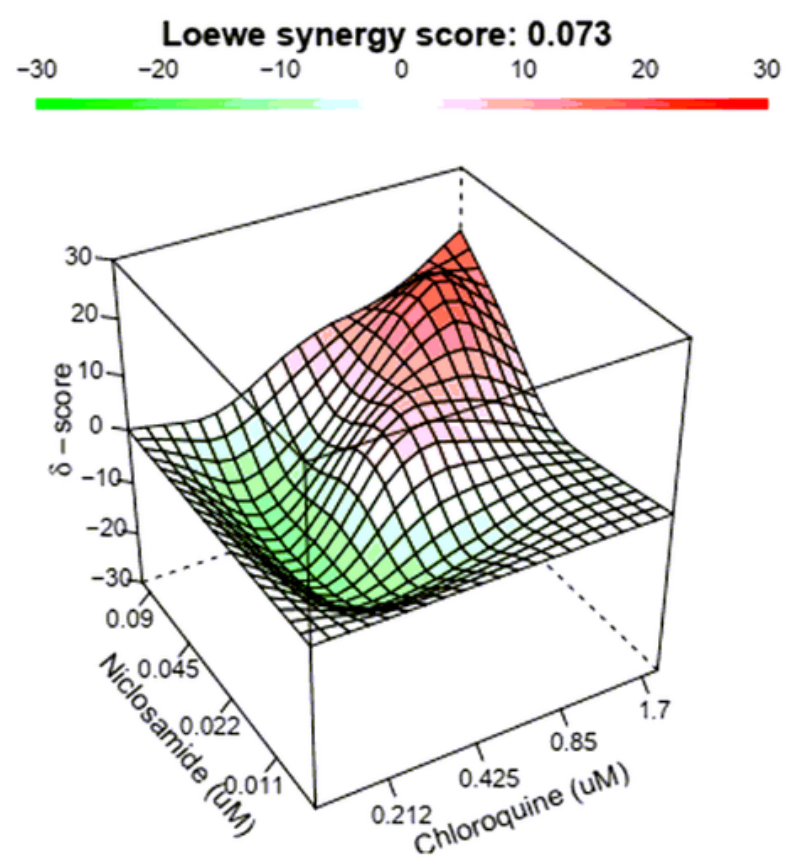


\section{Figure 3}

3Niclosamide-Chloroquine combination treatments against SARS-CoV-2 in Vero E6 cells. Vero E6 cells were treated for one hour with twofold serial dilutions of Niclosamide in the presence of different fixed concentrations of Chloroquine (A) or alternatively, serial dilutions of Chloroquine in the presence of different fixed concentrations of Niclosamide (B). Then the cells were infected with SARS-CoV-2 at m.o.i. 0.01. After removing the virus inoculum, the cells were further maintained in the medium containing drugs for 2 days. The viral RNA was determined using one-step qRT-PCR. The SynergyFinder was used to calculate the synergy score of two-drug combinations from different 16 pairwise combinations. The doseresponse matrix (C) and the synergy map of two-drug combinations treatment (D) were shown. The interaction landscape between two drugs was calculated using Loewe model. Areas with synergy score less than -10: the interaction between two drugs is likely to be antagonistic; from -10 to 10: the interaction between two drugs is likely to be additive; larger than 10: the interaction between two drugs is likely to be synergistic. The experiments were repeated at least three times, and data are shown as mean \pm SD. 
$\rightarrow$ Ivermectin

$\multimap$ Ivermectin+Chloroquine $1.7 \mu \mathrm{M}$

- Ivermectin+Chloroquine $0.85 \mu \mathrm{M}$

$\rightarrow$ Ivermectin+Chloroquine $0.425 \mu \mathrm{M}$

- Ivermectin+Chloroquine $0.2125 \mu \mathrm{M} \cdot \boldsymbol{\nabla} \cdot$

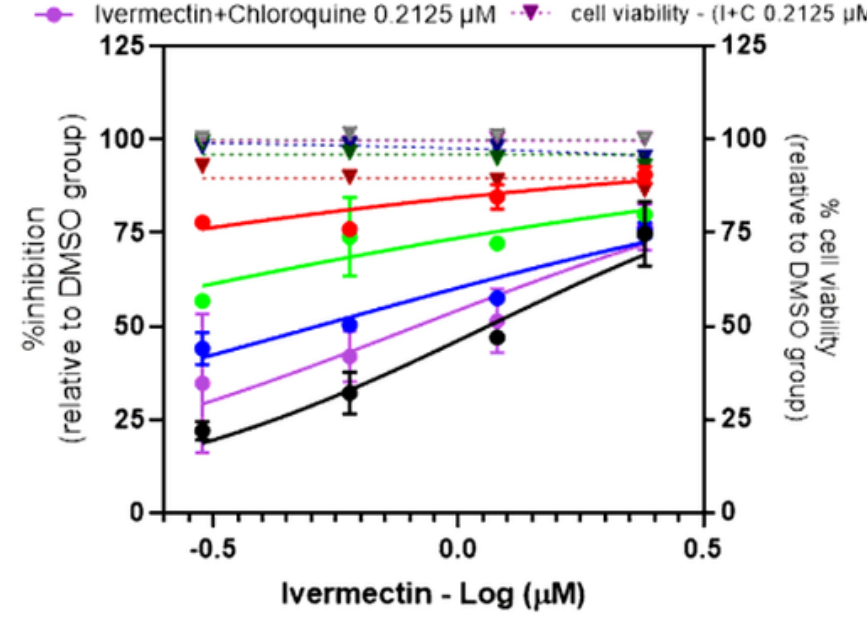

C

\section{Dose-response matrix (inhibition)}

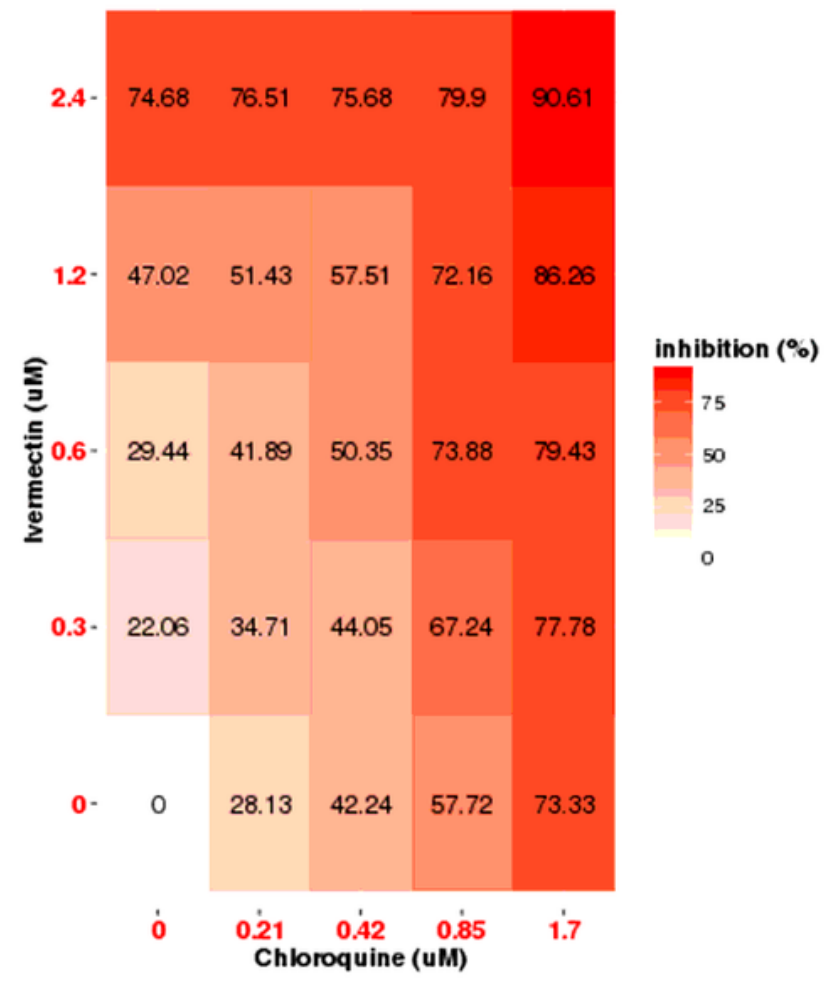

$\rightarrow$ Ivermectin

$\rightarrow$ Ivermectin+Chloroquine $1.7 \mu \mathrm{M} \quad \nabla \cdot$ cell viablity - (I)

- Ivermectin+Chloroquine $0.85 \mu \mathrm{M}$

$\rightarrow$ Ivermectin+Chloroquine $0.425 \mu \mathrm{M}$

7. cell viability $=(1+\mathrm{C} 1.7 \mu \mathrm{M})$

-. cell viability $-(1+C 0.85 \mu \mathrm{M})$

$\rightarrow$ Ivermectin+Chloroquine $0.2125 \mu \mathrm{M}-\boldsymbol{\nabla}$. cell viability $-(1+\mathrm{C} 0.2125 \mu \mathrm{M})$

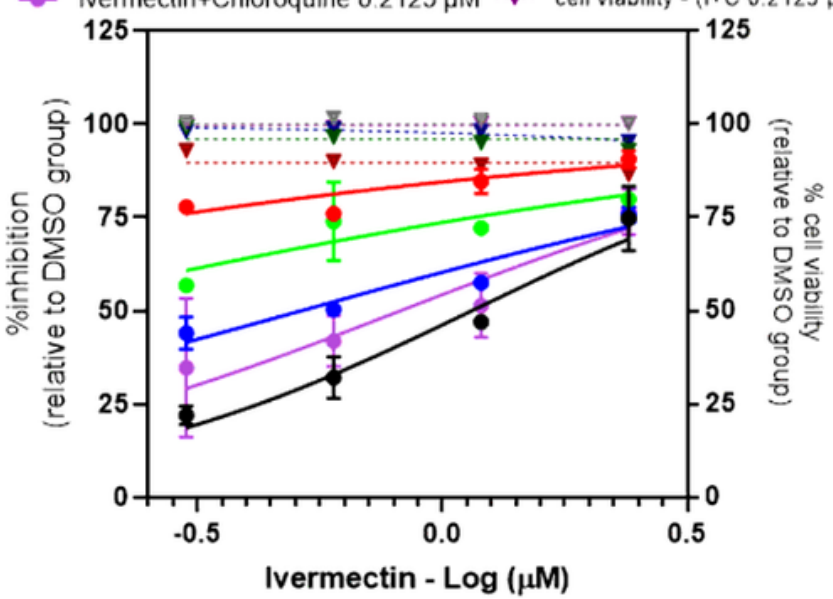

D
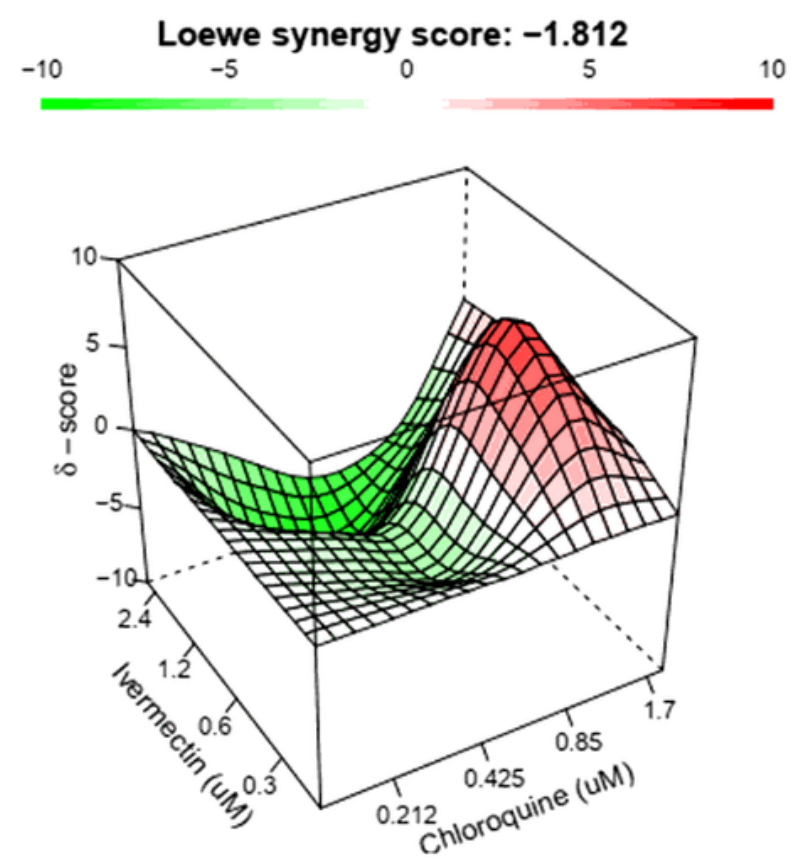

\section{Figure 4}

Ivermectin-Chloroquine combination treatments against SARS-CoV-2 in Vero E6 cells. Vero E6 cells were treated for one hour with twofold serial dilutions of Ivermectin in the presence of different fixed concentrations of Chloroquine $(A)$ or alternatively, serial dilutions of Chloroquine in the presence of different fixed concentrations of Ivermectin (B). Then the cells were infected with SARS-CoV-2 at m.o.i. 0.01. After removing the virus inoculum, the cells were further maintained in the medium containing drugs 
for 2 days. The viral RNA was determined using one-step qRT-PCR. The SynergyFinder was used to calculate the synergy score of two-drug combinations from different 16 pairwise combinations. The doseresponse matrix (C) and the synergy map of two-drug combinations treatment (D) were shown. The interaction landscape between two drugs was calculated using Loewe model. Areas with synergy score less than -10: the interaction between two drugs is likely to be antagonistic; from -10 to 10: the interaction between two drugs is likely to be additive; larger than 10: the interaction between two drugs is likely to be synergistic. The experiments were repeated at least three times, and data are shown as mean \pm SD.

A

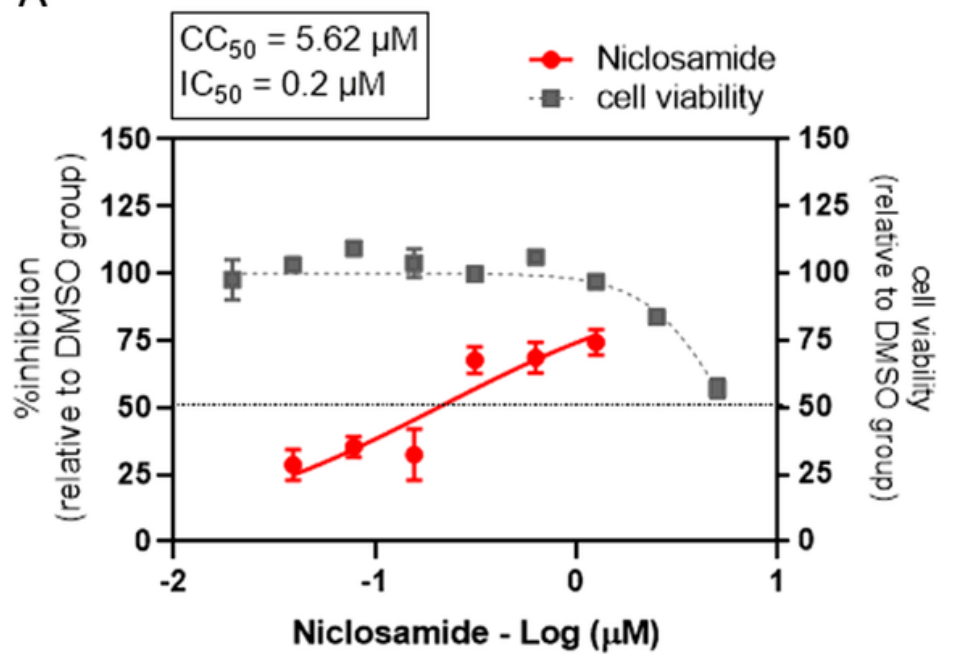

B

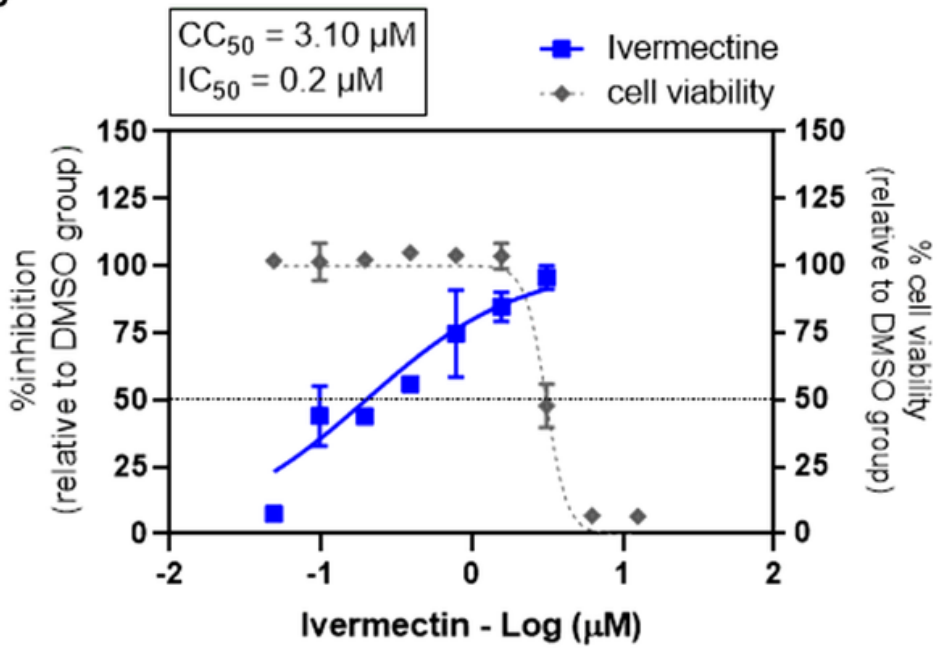

\section{Figure 5}

Single drug treatment against SARS-CoV-2 in Calu-3 cells The dose-response curves of a single drug treatment against SARS-CoV-2 are shown; (A) Niclosamide, and (B) Ivermectin. Calu-3 cells were treated with twofold serial dilutions of drug for one hour and infected with SARS-CoV-2. The infected cells were maintained in the medium containing serial dilutions of drugs or $0.5 \%$ DMSO for two days. Virus production was determined using a plaque assay. The dose-response curves are expressed as the percent inhibitions in relative to the DMSO-treated cell. The effect of drug treatment on cell viability was determined using MTT assay. 


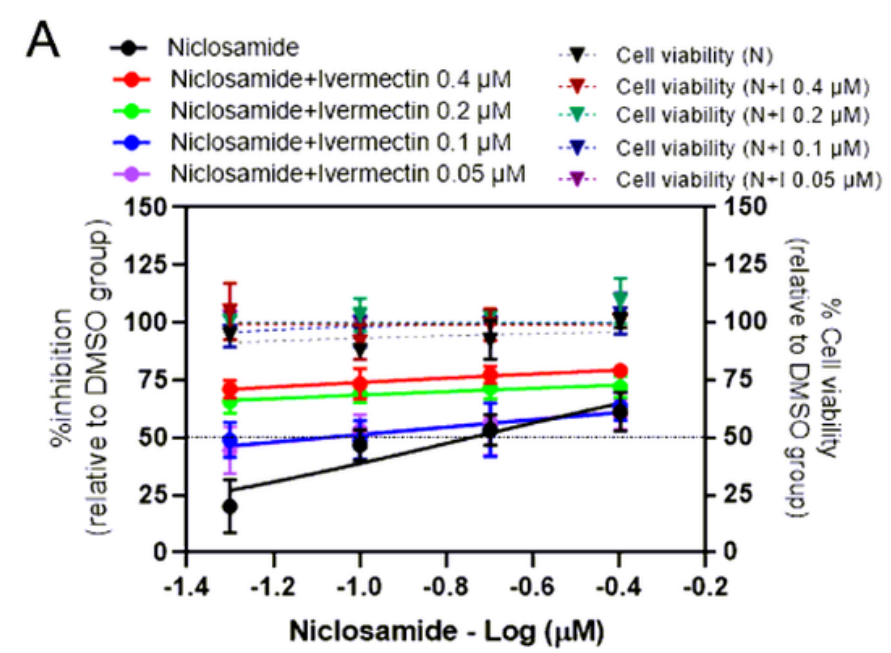

C Ivermectin \& Niclosamide

Dose-response matrix (inhibition)

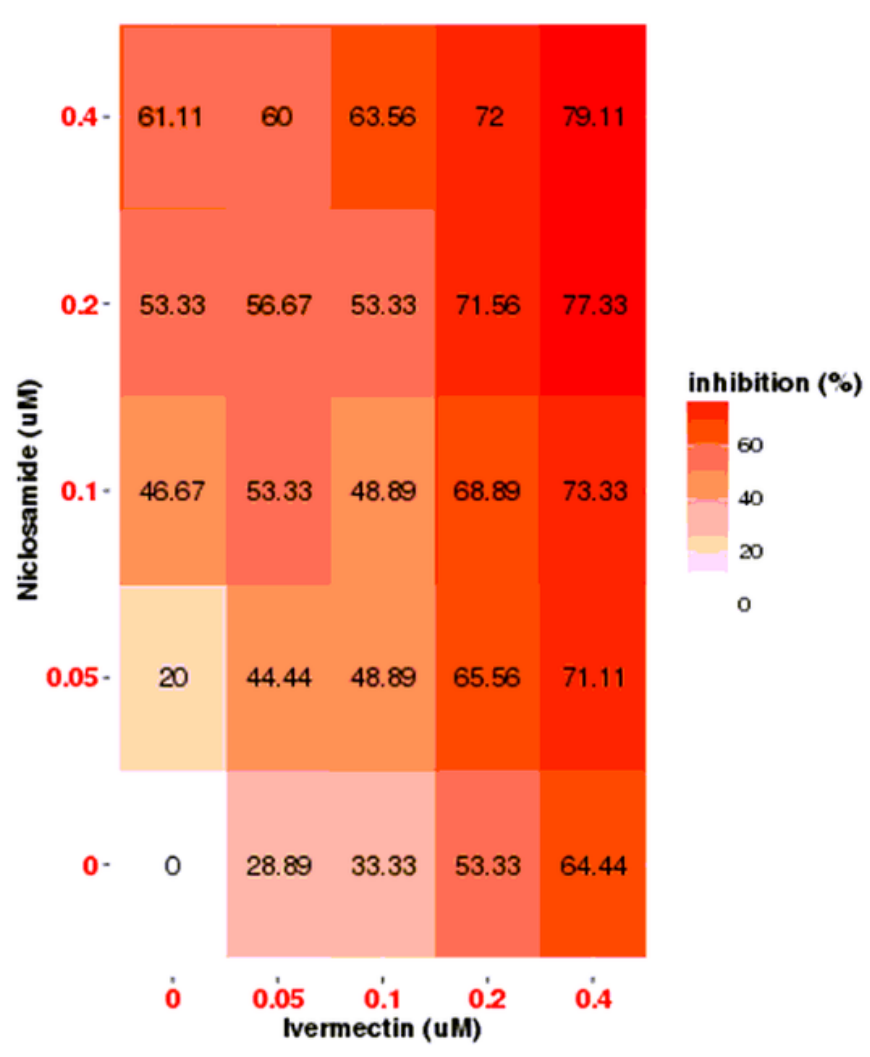

B

$\multimap$ Ivermectin
$\multimap$ Ivermectin+Niclosamide $0.4 \mu \mathrm{M}$
$\multimap$ Ivermectin+Niclosamide $0.2 \mu \mathrm{M}$
$\multimap$ Ivermectin+Niclosamide $0.1 \mu \mathrm{M}$

\&. Cell viability (I)

*. Cell viability $(1+\mathrm{N} 0.4 \mu \mathrm{M})$

- Cell viability $(\mathrm{I}+\mathrm{N} 0.2 \mu \mathrm{M})$

*. Cell viability $(\mathrm{I}+\mathrm{N} 0.1 \mu \mathrm{M})$

- Ivermectin+Niclosamide $0.05 \mu \mathrm{M}$

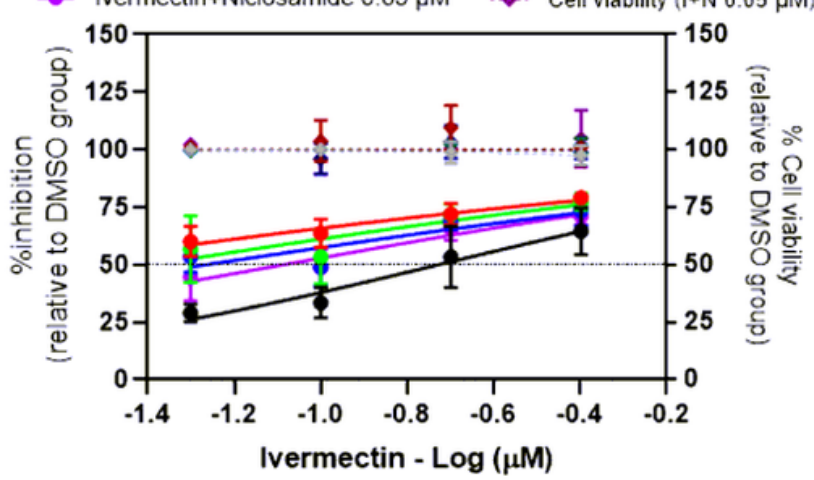

Ivermectin - $\log (\mu \mathrm{M})$

D
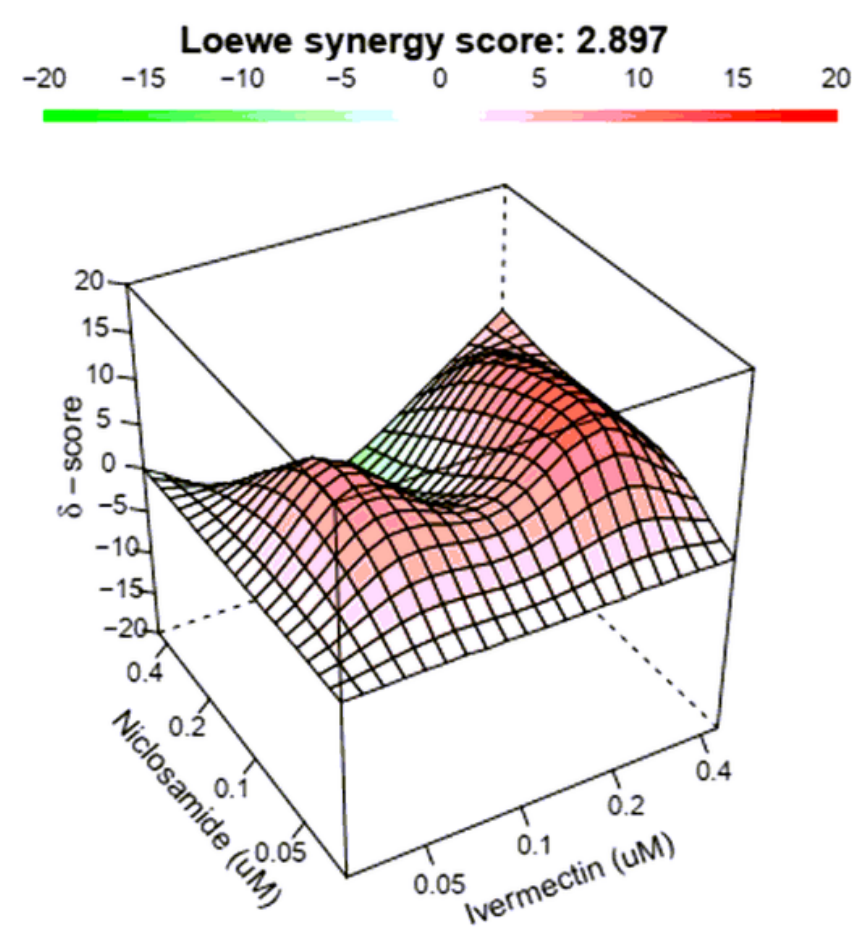

\section{Figure 6}

Niclosamide-Ivermectin combination treatments against SARS-CoV-2 in Calu-3 cells Calu-3 cells were treated for one hour with twofold serial dilutions of Niclosamide in the presence of different fixed concentrations of Ivermectin $(A)$ or alternatively, serial dilutions of Ivermectin in the presence of different fixed concentrations of Niclosamide (B). Then the cells were infected with SARS-CoV-2 at m.o.i. 0.01. After removing the virus inoculum, the cells were further maintained in the medium containing drugs for 2 days. The viral RNA was determined using one-step qRT-PCR. The SynergyFinder was used to calculate 
the synergy score of two-drug combinations from different 16 pairwise combinations. The dose-response matrix (C) and the synergy map of two-drug combinations treatment (D) were shown. The interaction landscape between two drugs was calculated using Loewe model. Areas with synergy score less than -10: the interaction between two drugs is likely to be antagonistic; from -10 to 10: the interaction between two drugs is likely to be additive; larger than 10: the interaction between two drugs is likely to be synergistic. The experiments were repeated for three times, and data are shown as mean \pm SD. 